\title{
PROOF OF THE FUNDAMENTAL THEOREMS ON SECOND-ORDER CROSS PARTIAL DERIVATIVES*
}

BY

A. E. CURRIER

1. Introduction. In the present article we prove the following theorem:

Let $f(x, y)$ be defined on an open region $R$, and let the first partial derivatives $f_{x}$ and $f_{y}$ exist on $R$. Let $A$ be a point set on which the four second-order partial derivatives

$$
f_{x x}, f_{x y}, f_{y x}, f_{y y}
$$

exist almost everywhere. Then

$$
f_{x y}=f_{y x}
$$

almost everywhere on $A$.

At the conclusion of this paper we state a number of interesting problems which are closely connected with the theorems which we prove. We wish to thank Dr. S. Saks for many helpful suggestions.

2. Measurability of second partial derivatives. We require the following lemma.

Lemma 1. Let $u(x, y)$ be a function of. Baire, and let $A_{n}$ be the point set on which the following inequality is satisfied:

$$
\frac{u(x+h, y)-u(x, y)}{h}<\alpha, \quad 0<h<\frac{1}{n},(x, y)<A_{n} .
$$

Then $A_{n}$ is measurable.

We readily see that the function

$$
\frac{u(x+h, y)-u(x, y)}{h}
$$

is a function of Baire in the space of the three variables $(x, y, h)$. Let $\Delta$ be the portion of the space $(x, y, h)$ for which $0<h<1 / n$. Let $\mathfrak{A}_{n}$ be the portion of $\Delta$ for which the inequality (2.1) is satisfied. We see that the complement of the

* Presented to the Society, December 27, 1932; received by the editors, May 20, 1932, and, in revised form, June 8, 1932. 
set $A_{n}$ of Lemma 1 is obtained by projecting $\Delta-\mathfrak{A}_{n}$ onto the space $(x, y)$. Thus $A_{n}$ is a complementary analytic set, and hence measurable.*

From Lemma 1 the reader will readily see that the following lemma is true.

LEMMA 2. Let $f(x, y)$ be a function of Baire, defined on an open region $R$. Let the first partial derivative $f_{x}$ exist on $R$. Then the second partial derivatives $f_{x x}$ and $f_{x y}$ are measurable functions on their respective domains of definition.

3. Cross sections of a closed point set. We make the following definition.

Definition. If $P$ is a closed point set in the plane $(x, y)$ then $P_{x}(t)$ and $P_{y}(t)$ will denote the cross sections of $P$ with the lines $x=t$ and $y=t$ respectively.

We shall require the following lemma.

Lemma 3. If $P$ is closed $\dagger$ then almost every point $\left(x_{0}, y_{0}\right)$ of $P$ is a density point in the linear sense of the cross sections $P_{x}\left(x_{0}\right)$ and $P_{y}\left(y_{0}\right)$, and in the superficial sense a density point of $P$.

We denote by $\phi(x, y)$ the characteristic function $\ddagger$ of $P$ and set

$$
\psi(x, y)=\int_{0}^{y} \phi(x, y) d y .
$$

By a well known theorem the partial derivative $\psi_{\nu}$ exists and equals $\phi(x, y)$ almost everywhere in the plane. That is, $\psi_{y}(x, y)=1$ almost everywhere on $P$. This shows that almost every point of $P$ is a density point in the linear sense of the corresponding cross section $P_{x}(x)$. A similar proof applies to the cross sections $P_{v}(y)$. It is well known that the superficial density points of $P$ lie almost everywhere on $P$, hence Lemma 3 is correct.

4. The approximate middle derivative. The middle difference quotient $\Delta f / \lambda^{2}$ of a function $f(x, y)$ at the point $\left(x_{0}, y_{0}\right)$ is defined as follows:

$$
\frac{\Delta f}{\lambda^{2}}=\frac{f\left(x_{0}+\lambda, y_{0}+\lambda\right)-f\left(x_{02} y_{0}+\lambda\right)-f\left(x_{0}+\lambda, y_{0}\right)+f\left(x_{0}, y_{0}\right)}{\lambda^{2}} \text {. }
$$

The approximate middle derivative of $f(x, y)$ at $\left(x_{0}, y_{0}\right)$ (when it exists) will be defined as the following approximate limit\$:

* N. Lusin, Sur les ensembles analytiques, Fundamenta Mathematicae, vol. 10 (1927), pp. 1-95, especially pp. 25-26. Also Lusin et Sierpinski, Sur quelques propriétés des ensembles $(A)$, Bulletin de l'Académie de Cracovie, 1918, p. 44.

† The lemma holds if $P$ is merely measurable.

$\ddagger$ The characteristic function of a point set equals one on points of the set and equals zero elsewhere.

§ Cf. Lebesgue, Leçons sur l'Intégration, Paris, 1928, pp. 240-241, for a definition of approximate limits. 


$$
\operatorname{approx}-D_{m} f=\operatorname{approx}-\lim _{\lambda \rightarrow 0} \frac{\Delta f}{\lambda^{2}}
$$

5. The fundamental lemma. We now state the fundamental lemma as follows.

Fundamental Lemma. Let $f(x, y)$ be a function of Baire defined on an open region $R$, and let the first partial derivative $f_{x}(x, y)$ exist on $R$. Let $A$ be the subset of $R$ on which the partial derivatives

$$
f_{x x}, f_{x y}
$$

exist and take on finite values. Then*

$$
\text { approx- } D_{m} f=f_{x y}
$$

almost everywhere on $A$.

The functions $f_{x x}$ and $f_{x y}$ are measurable by Lemma 2 , hence the set $A$ is measurable. Let $A_{n}$ be the part of $A$ for which the following inequalities are satisfied:

$$
\begin{aligned}
& \left|\frac{f_{x}(x+h, y)-f_{x}(x, y)}{h}\right|<n, \\
& \left|\frac{f_{x}(x, y+k)-f_{x}(x, y)}{k}\right|<n,
\end{aligned}
$$

for $(x, y)<A_{n}$ and $0<|h|,|k|<1 / n$.

We readily see that the sets $A_{n}$ cover the set $A$. Hence in order to prove the Fundamental Lemma it is sufficient to show that (5.1) holds almost everywhere on the set $A_{n}$.

By Lemma 1 the set $A_{n}$ is seen to be measurable, and hence by a well known theorem there exists a sequence $\left\{P_{k}\right\}$ of closed parts of $A_{n}$ which cover $A_{n}$ almost everywhere. Hence in order to prove the Fundamental Lemma it is sufficient to show that (5.1) holds almost everywhere on each closed part of $A_{n}$.

Let $P$ be a closed part of $A_{n}$. From (5.3) it follows that the function $f_{x y}$ is bounded on $P$. Since $f_{x y}$ is measurable and bounded on $P$ it is summable on $P$ and by a well known theorem for almost every point $\left(x_{0}, y_{0}\right)$ of $P$

$$
\lim _{\lambda \rightarrow 0} \frac{1}{\lambda^{2}} \int_{P \delta} f_{x y} d x d y=f_{x y}\left(x_{0}, y_{0}\right)
$$

* Equation (5.1) implies the existence of approx- $D_{m} f$, that is, approx- $D_{m} f$ exists and equals $f_{x y}$ almost everywhere on $A$. 
where $\delta$ is the square with one corner at the point $\left(x_{0}, y_{0}\right)$ and the opposite corner at the point $\left(x_{0}+\lambda, y_{0}+\lambda\right)$. Moreover by Lemma 3 almost every point $\left(x_{0}, y_{0}\right)$ of $P$ is a density point in the linear sense of the cross sections of $P$ and in the superficial sense a density point of $P$. Hence in order to prove that (5.1) holds almost everywhere on $P$ it is sufficient to show that (5.1) holds for the point $\left(x_{0}, y_{0}\right)$ of $P$, where $\left(x_{0}, y_{0}\right)$ is a density point of $P$ in the linear and superficial senses and is also a point for which (5.4) holds. Thus we see that in order to prove the Fundamental Lemma it is sufficient to prove the following auxiliary lemma.

Auxiliary Lemma. Let the hypotheses of the Fundamental Lemma be satisfied. Let $P$ be a closed part of $A$ such that the inequalities (5.2) and (5.3) are satisfied for $(x, y)$ on $P$.

Let $\left(x_{0}, y_{0}\right)$ be a density point in the linear sense of the cross sections of $P$ and in the superficial sense a density point of $P$. Moreover let $\left(x_{0}, y_{0}\right)$ be a point of $P$ at which equation (5.4) holds.

Then approx- $D_{m} f$ exists at $\left(x_{0}, y_{0}\right)$ and equals $f_{x y}\left(x_{0}, y_{0}\right)$.

6. Proof of the Auxiliary Lemma. Let $e$ be the set of values of $\lambda$ corresponding to which the points $\left(x_{0}, y_{0}+\lambda\right)$ lie in $P$. We see that the point $\lambda=0$ is a density point of $e$, since $\left(x_{0}, y_{0}\right)$ is a density point in the linear sense of the cross section $P_{x}\left(x_{0}\right)$ of $P$. For $\lambda$ in $e$ and constant we see by (5.2) that $f_{x}\left(x, y_{0}+\lambda\right)$ is uniformly bounded for $\left|x-x_{0}\right|<1 / n$. Hence the middle increment $\Delta f$ at $\left(x_{0}, y_{0}\right)$ can be expressed by means of an integral as follows:

$$
\Delta f=\int_{x_{0}}^{x_{0}+\lambda}\left[f_{x}\left(x, y_{0}+\lambda\right)-f_{x}\left(x, y_{0}\right)\right] d x .
$$

As before, let $\delta$ be the closed square with one corner at $\left(x_{0}, y_{0}\right)$ and the opposite corner at $\left(x_{0}+\lambda, y_{0}+\lambda\right)$. Let $e_{1}$ be the projection of $P \delta$ on the $x$-axis, and let $e_{2}$ be the complement of $e_{1}$ with respect to the closed interval $x_{0} \leqq x \leqq$ $x_{0}+\lambda$ (or $x_{0}+\lambda \leqq x \leqq x_{0}$ if $\lambda<0$ ).

Let $O$ denote the ordinate which passes through the point $(x, 0)$. Then the product set $O P$ is the cross section $P_{x}(x)$ of $P$. The set $O P \delta$ is closed and nonempty for $x$ in $e_{1}$. The complementary set $O \delta-O P \delta$ is open on $O \delta$ and consists of at most a denumerable infinity of open linear intervals on the ordinate $O$. Let $(x, \alpha)$ and $(x, \beta)$ be the end points of a general one of these intervals. In the difference quotient

$$
\frac{f_{x}(x, \beta)-f_{x}(x, \alpha)}{\beta-\alpha}
$$

at least one of the points $(x, \alpha),(x, \beta)$ is a point of $P$. Moreover $|\beta-\alpha|<|\lambda|$. 
Hence by (5.3) we have

$$
\left|f_{x}(x, \beta)-f_{x}(x, \alpha)\right|<n|\beta-\alpha|,|\lambda|<\frac{1}{n}
$$

Hence for $x$ in $e_{1}$ and $|\lambda|<1 / n$ the difference $f_{x}\left(x, y_{0}+\lambda\right)-f_{x}\left(x, y_{0}\right)$ can be expressed as follows:*

(6.3) $f_{x}\left(x, y_{0}+\lambda\right)-f_{x}\left(x, y_{0}\right)=\int_{O P \delta} f_{x y}(x, y) d y+\sum_{\alpha, \beta}\left[f_{x}(x, \beta)-f_{x}(x, \alpha)\right]$.

Comparing (6.1) and (6.3) we see that for $\lambda$ in $e$ and $|\lambda|<1 / n$ the middle increment $\Delta f$ can be expressed in the form

$$
\Delta f=\int_{e_{1}} d x \int_{O P \delta} f_{x y}(x, y) d y+\int_{e_{1}} \sum_{\alpha, \beta}\left[f_{x}(x, \beta)-f_{x}(x, \alpha)\right] d x
$$

$$
+\int_{e_{2}}\left[f_{x}\left(x, y_{0}+\lambda\right)-f_{x}\left(x, y_{0}\right)\right] d x \text {. }
$$

As remarked above the point $\lambda=0$ is a density point of $e$. Hence in order to prove the Auxiliary Lemma it is sufficient to show that

$$
\lim _{\lambda \rightarrow 0} \frac{\Delta f}{\lambda^{2}}=f_{x y}\left(x_{0}, y_{0}\right)
$$

where the notation on the left indicates that $\lambda$ is to approach zero through values which lie in $e$.

The first integral on the right of (6.4) is merely the double integral of $f_{x y}(x, y)$ taken over $P \delta$. Because of (5.4) we see that in order to prove (6.5) it is sufficient to show that the following equations are true:

$$
\begin{aligned}
& \lim _{\lambda \rightarrow 00} \frac{1}{\lambda^{2}} \int_{e_{1}} \sum_{\alpha, \beta}\left[f_{x}(x, \beta)-f_{x}(x, \alpha)\right] d x=0, \\
& \lim _{\lambda \rightarrow 0} \frac{1}{\lambda^{2}} \int_{e_{2}}\left[f_{x}\left(x, y_{0}+\lambda\right)-f_{x}\left(x, y_{0}\right)\right] d x=0 .
\end{aligned}
$$

Because of (5.3) we see that the absolute value of the sum in the integrand (6.6) is bounded by

$$
n \sum_{\alpha, \beta}|\beta-\alpha|=n \mu(O \delta-O P \delta)
$$

* Cf. Lebesgue, loc. cit., pp. 210-211. The series in (6.3) is readily seen to be absolutely convergent by (6.2). 
where $\mu$ denotes linear Lebesgue measure on the ordinate $O$. Hence the absolute value of the expression (6.6) is bounded by the following expression:

(6.8) $\frac{1}{\lambda^{2}} \int_{e_{1}} n \mu(O \delta-O P \delta) d x \leqq \frac{1}{\lambda^{2}} \int_{x_{0}}^{x_{0}+\lambda} n \mu(O \delta-O P \delta) d x=n \frac{m(\delta-P \delta)}{\lambda^{2}}$,

where $m$ denotes superficial Lebesgue measure. Since $\left(x_{0}, y_{0}\right)$ is a superficial density point of $P$ the expression on the right of (6.8) converges to zero with $\lambda$. Hence (6.6) is correct.

To prove (6.7) we rewrite the integrand as follows:

$$
\begin{aligned}
f_{x}\left(x, y_{0}+\lambda\right) & -f_{x}\left(x, y_{0}\right)=f_{x}\left(x, y_{0}+\lambda\right)-f_{x}\left(x_{0}, y_{0}+\lambda\right) \\
& +f_{x}\left(x_{0}, y_{0}+\lambda\right)-f_{x}\left(x_{0}, y_{0}\right)+f_{x}\left(x_{0}, y_{0}\right)-f_{x}\left(x, y_{0}\right) .
\end{aligned}
$$

Thus we see that in order to prove (6.7) it is sufficient to show that the following three equations hold:

$$
\begin{aligned}
& \lim _{\lambda \rightarrow e_{0}} \frac{1}{\lambda^{2}} \int_{e_{2}}\left[f_{x}\left(x, y_{0}+\lambda\right)-f_{x}\left(x_{0}, y_{0}+\lambda\right)\right] d x=0 ; \\
& \lim _{\lambda \rightarrow e_{0} 0} \frac{1}{\lambda^{2}} \int_{e_{2}}\left[f_{x}\left(x_{0}, y_{0}+\lambda\right)-f_{x}\left(x_{0}, y_{0}\right)\right] d x=0 ; \\
& \lim _{\lambda \rightarrow e_{e} 0} \frac{1}{\lambda^{2}} \int_{e_{2}}\left[f_{x}\left(x_{0}, y_{0}\right)-f_{x}\left(x, y_{0}\right)\right] d x=0 .
\end{aligned}
$$

For $\lambda$ in $e$ the point $\left(x_{0}, y_{0}+\lambda\right)$ lies in $P$ and for $\lambda$ in $e$ and $|\lambda|<1 / n$ we see from (5.2) that the absolute value of the expression (6.10) is bounded by

$$
\frac{1}{\lambda^{2}}|\lambda| n \nu e_{2}=n\left|\frac{\nu e_{2}}{\lambda}\right|,
$$

where $\nu$ denotes linear Lebesgue measure in the $x$-space. From the fact that $\left(x_{0}, y_{0}\right)$ is a density point in the linear sense of the cross section $P_{y}\left(y_{0}\right)$ of $P$ we readily see that the expression on the right of (6.13) converges to zero as $\lambda$ approaches zero. Hence (6.10) is correct. In a similar way we prove that (6.11) and (6.12) are correct. Hence the Auxiliary Lemma and the Fundamental Lemma of $\$ 5$ are correct.

7. The first theorem on second-order partial derivatives. We now prove the following theorem.

THEOREM 1. Let $f(x, y)$ be a function of Baire defined on an open region $R$, and let the first partial derivative $f_{x}(x, y)$ exist on $R$. Let $A$ be a point set on which the partial derivatives $f_{x x}$ and $f_{x y}$ exist almost everywhere. Then the approximate middle derivative approx- $D_{m} f$ exists almost everywhere on $A$ and 


$$
\operatorname{approx}-D_{m} f=f_{x y}
$$

almost everwhere on $A$.

Let $A^{\prime}$ be the part of $A$ on which $f_{x x}$ and $f_{x y}$ exist and take on finite values. The set $A-A^{\prime}$ has superficial measure zero, ${ }^{*}$ hence in order to prove Theorem 1 it is sufficient to show that (7.1) holds almost everywhere on $A^{\prime}$. Let $A^{\prime \prime}$ be the set on which $f_{x x}$ and $f_{x y}$ exist and take on finite values. The set $A^{\prime}$ is part of $A^{\prime \prime}$. By the Fundamental Lemma approx- $D_{m} f$ exists and equals $f_{x y}$ almost everywhere on $A^{\prime \prime}$, hence almost everywhere on $A^{\prime}$. Thus Theorem 1 is correct.

8. Equality of the cross partial derivatives. We now prove the following:

THEOREM 2. Let $f(x, y)$ be defined on an open region $R$, and let the first partial derivatives $f_{x}(x, y)$ and $f_{y}(x, y)$ exist on $R$. Let $A$ be a point set on which the four second-order partial derivatives $f_{x x}, f_{x y}, f_{y x}, f_{y y}$ exist almost everywhere. Then $f_{x y}=f_{y x}$ almost everywhere on $A$.

Since $f_{x}$ and $f_{y}$ exist, the function $f(x, y)$ is continuous in $x$ alone and continuous in $y$ alone, and is thus a function of Baire. By Theorem 1 approx- $D_{m} f$ $=f_{x y}$ almost everywhere on $A$. By reasoning similar to the proof of Theorem 1 we see that approx $-D_{m} f=f_{y x}$ almost everywhere on $A$. That is

$$
f_{x y}=\operatorname{approx}-D_{m} f=f_{y x}
$$

almost everywhere on $A$. Thus Theorem 2 is seen to be true.

9. Generalizations of Theorems 1 and 2. In Theorems 1 and 2 it is not necessary to assume that the first derivatives exist. We make the following definition:

DEFINITION 1. Let $f(x, y)$ be an arbitrary function, and let

$$
\bar{f}_{x}+(x, y), \underline{f}_{x}+(x, y), \bar{f}_{x}^{-}(x, y), \underline{f}_{x}^{-}(x, y)
$$

be the four principal first partial derivatives $\dagger$ of $f(x, y)$ with respect to $x$. The second partial derivative $f_{x x}$ is said to exist at $\left(x_{0}, y_{0}\right)$ if the first derivative $f_{x}\left(x_{0}, y_{0}\right)$ exists (that is, if the four functions (9.1) have the same value at $\left(x_{0}, y_{0}\right)$ ) and if the four functions (9.1) are partially differentiable with respect to $x$ at $\left(x_{0}, y_{0}\right)$ and have the same value for their partial derivative with respect to $x$ at $\left(x_{0}, y_{0}\right)$.

* The set $A-A^{\prime}$ consists of the part of $A$ on which $f_{x x}$ or $f_{x y}$ does not exist (this part is of measure zero) plus the part of $A$ on which either $f_{x x}$ or $f_{x y}$ is infinite. The latter part also has measure zero. The function $f_{x x}$ is measurable, and the linear measure of each cross section of the part of $A$ on which $f_{x x}= \pm \infty$ is zero, as proved for example in Hobson, Theory of Functions of a Real Variable, Cambridge, 1927, vol. I, p. 397, Theorem 2. Similarly for infinite values of $f_{x y}$.

† Cf. Carathéodory, Vorlesungen über reelle Funktionen, Berlin, 1927, p. 641. 
We make similar definitions for the existence of the remaining second partial derivatives. We now state certain generalizations of Theorems 1 and 2.

THEOREM 3. Let $f(x, y)$ be defined on an open region $R$, and be continuous in $x$ alone and in $y$ alone. Let $D_{x}$ be one of the principal first partial derivatives (9.1). Let $A$ be a point set on which the partial derivatives

$$
\frac{\partial}{\partial x} D_{x} f, \frac{\partial}{\partial y} D_{x} f
$$

exist almost everywhere. Then

$$
\text { approx- } D_{m} f=\frac{\partial}{\partial y} D_{x} f
$$

almost everywhere on $A$.

It is well known that under the hypotheses of Theorem 3 the function $D_{x} f$ is a function of Baire. The proof of Theorem 3 now follows in the same way as the proof of Theorem 1, after a suitable restatement and reproof of the Fundamental Lemma. No new methods are required.

Theorem 4. Let $f(x, y)$ be defined on an open region $R$, and let $f(x, y)$ be continuous in $x$ alone and in $y$ alone. Let $A$ be a point set on which the second partial derivatives

$$
f_{x x}, f_{x y}, f_{y x}, f_{y y}
$$

exist in the generalized sense almost everywhere. Then

$$
f_{x y}=f_{y x}
$$

almost everywhere on $A$.

The proof of this theorem follows readily, making use of Theorem 3.

10. Problems. Certain problems present themselves at once. We state a few of them here.

Problem 1. Let $f(x, y)$ be a function of Baire. Are the principal first partial derivative functions (9.1) also functions of Baire*?

If the answer to Problem 1 is in the affirmative the following theorem follows at once by the methods of this paper.

* For a function $f(x)$ of one variable the answer is in the affirmative; cf. Sierpinski, Sur les fonctions dérivées des fonctions discontinues, Fundamenta Mathematicae, vol. 3 (1922), pp. 123-127. In case $f(x, y)$ is continuous in $x$ the answer is in the affirmative. 
Let $f(x, y)$ be a function of Baire. Let $A$ be a point set on which the second partial derivatives $f_{x x}, f_{x y}, f_{y x}, f_{y y}$ exist in the generalized sense almost everywhere. Then $f_{x y}=f_{y x}$ almost everywhere on $A$.

Problem 2. Let $f(x, y)$ together with its first partial derivatives $f_{x}$ and $f_{y}$ be continuous on an open region $R$. Let $A$ be a point set on which $f_{x x}$ and $f_{x y}$ exist almost everywhere. Then we know that approx- $D_{m} f$ exists and equals $f_{x y}$ almost everywhere on $A$. Does the actual middle derivative $D_{m} f$ exist almost everywhere on $A$ ?

Problem 3. Is Theorem 1 true if $f(x, y)$ is merely measurable?

It is probable that an example can be constructed which will show that the answer to this problem is in general negative.

A large number of related problems can be readily thought of, problems which have to do with the reversal of the order of integration in iterated integrals, and problems connected with two-dimensional totalization. It is of course quite obvious that theorems similar to Theorems 1, 2, 3, and 4 can be stated concerning the third partial derivatives $f_{x y z}$ etc. of a function $f(x, y, z)$ of three variables, and corresponding general theorems for the partial derivatives of functions of $n$ variables.

HARVARD UNIVERSITY,

Cambridge, Mass. 